\title{
The assessment of vision in the preschool child
}

\author{
Patricia M Sonksen
}

The developmental, optometric, and practical issues that govern clinical examination of vision from birth to 5 years and their bearing upon diagnostic and surveillance practice is discussed.

Our current understanding is that minor defects of the visual system do not significantly constrain general developmental progress or early visual development while severe visual impairment can seriously impede both. ${ }^{1-3}$ Both benefit from skilled developmental management. ${ }^{34}$ The earlier surgical and optical treatment are implemented for the few conditions amenable to this approach such as cataract, retinoblastoma, and glaucoma, the greater the benefits in terms of vision, general development, and health. At least two thirds of babies with severe visual impairment require paediatric management including genetic counselling; the effectiveness of this is improved by early diagnosis.

\section{Visual behaviours}

The net to catch babies medically and/or genetically at risk of severe visual impairment are well summarised in the recommendations of the Joint Working Party on Health for All Children. ${ }^{5}$ These highlight the physical examination of the visual system and the importance of parental concern about eyes or visual behaviour. However, the elicitation of early visual behaviours, such as fixation and following of a $6.5 \mathrm{~cm}$ ball or fixation of tiny round objects in near and distance, are given scant credence. This is a pity because methods that evaluate vision, in addition to a physical examination of the eyes, are required in the context of parental or medical concern about vision. The reason for what I see as premature dismissal of visual behaviours are twofold: misconception of their capability and imprecise specification for their scientific framework.

The two ghosts that need to be laid are first, that tests of visual behaviour exclude minor visual errors that may lead to later medical problems or interfere with later learning and second that they measure acuity. Tests of early visual behaviour are qualitative and only exclude severe visual impairment; they do not exclude minor problems such as hypermetropia or anisometropia of a degree likely to progress to squint and amblyopia. An individual may be visually aware of a single 'hundred and thousand' from $25 \mathrm{~cm}$ with acuity levels equivalent to normal peripheral vision and many with near acuity of N48 may fixate one. Acuity by definition requires resolution of adjacent visual targets whereas tests like the hundreds and thousands test only require detection of a single item. The confusion between acuity and detection vision probably arose because normal individuals use macular pathways for both and both are characterised by fixation.

To date, scientific specification of tests of visual behaviour has been extremely loose. Physical parameters of test materials, optical conditions, and the qualitative and quantitative aspects of pass/fail criteria are poorly defined. There is poor appreciation of the developmental rationale that determines test design, the aspect of vision being evaluated, and the developmental context in which findings are considered. ${ }^{6} \mathrm{By}$ way of example the following are a few of the many omissions in formatting the hundreds and thousands test. The optical rationale of using round objects such as a hundred and thousand (round cake decoration, diameter $1 \mathrm{~mm}$ ) rather than sugar strands $(1 \times 5$ to $10 \mathrm{~mm})$ is not explained; the effective size of a stimulus is determined by the greater dimension. Similarly, the optical relevance of using one rather than scattering many is neglected; moving objects stimulate 'streaks' rather than 'points' of the retina, abutting stationary targets effect a larger one and multiple targets impede the testers ability to confirm fixation. The position of the tester relative to the child is not rationalised and usually left to personal preference; a tester needs to be in front of and at, or just below, a child's eye level in order to monitor eye movements precisely. Reliable methods of masking placement, of creating standard levels of contrast between item and background and of eliminating sound cues are rarely detailed. Pass/fail criteria are too broad, one of the commonest being 'pokes at' or 'picks up' sweet between finger and thumb; developmental parameters other than vision (cognitive and fine motor development) are embodied in such criteria. The pass/fail criterion should be specific to vision - sharp fixation (direct pupil to object gaze) from a distance of at least $25 \mathrm{~cm}$. Any quality of gaze such as searching, peering, eccentric or poorly sustained fixation is abnormal and requires specialist assessment. Once the visual end point has been observed the developmental parameters alluded to above can, with advantage, be noted and recorded in appropriate subsections of the general assessment. The findings of a behavioural test are best interpreted in the light of developmental rather than chronological age. For example, if language and nonverbal levels are age appropriate in a 9 month old 
who fixates a Smartie $(1.25 \mathrm{~cm}$ round sweet $)$ but does not look at a hundred and thousand, the problem is more likely to be visual than if the former are at a 6 month level, when learning disability becomes the greater probability.

Tests of visual behaviours have, with some justification, been the subject of adverse criticism in recent times. Apart from lack of proper specification they were taken out of context of the rest of the visual examination and expected to stand in the face of criteria laid down for screening tests. ${ }^{7}$ We do not expect listening to heart sounds to screen for all forms of heart disease. Clinicians need to be able to examine vision as well as eyes and I am convinced that a carefully selected and formatted set of tests of visual behaviour would enhance paediatric and diagnostic services.

Occlusion provides an opportunity to explore the vision of each eye separately; age related factors such as the development of attention control and behaviour make success the exception rather than rule between 12 and 48 months of age. Between 7 and 12 months a baby's attention can be distracted from the 'hateful' patch to another focus; it makes developmental sense to advise surveillance practice to take this opportunity to examine for asymmetry in the quality of gaze of the two eyes. A graded series of small round sweets - Smartie, silver ball cake decoration ( $4 \mathrm{~mm})$, and a hundred and thousand - is sufficiently motivating to attract attention away from the patch and thus exclude a serious defect of vision in one eye only.

Most behavioural tests of vision can be adapted so that the findings provide a basis for prescription of programmes that will promote visual development and use residual vision optimally to enhance numerous aspects of general development in severely visually impaired babies. ${ }^{389}$

\section{Squint}

The prevalence of squint rises slowly from infancy to 7 years with a mean of about $3.5 \%$ for all ages; it is much higher in disabled populations. The significance, assessment, and management of different types of squint is well reviewed by Fielder. ${ }^{10}$ Squint is a feature of many disturbances of the visual system, both major and minor. It may predispose to, coexist with, or be the consequence of defective vision; on the other hand it also occurs in individuals with normal acuity. It is therefore essential that surveillance practice includes both an examination of vision (visual behaviour/acuity) and for squint. Performing tests for squint in babies and young children is not as easy as the instructions make them sound. The reasons are develop-

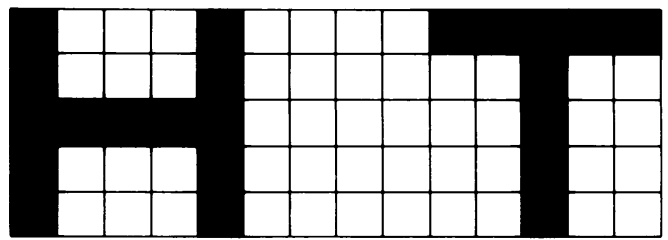

Integral proportions and spacing between optotypes according to the international standard. mental. For example, in the cover test the examiners 'covering' hand not only attracts the child's attention away from the visual lure but holds it because, not understanding its purpose, the child perceives it as a threat. Similarly, failure to understand that the examiner wishes to observe eye, as opposed to head, movements in concordance of gaze and light reflections tests leads to attempts at physical restraint and behavioural disaster. Strategies to circumvent these developmental constraints upon test success have grown with the experience of orthoptists, ophthalmologists, and developmental paediatricians. Examples are acclimatisation to the examiner's hand before introduction of a visual target, stationary targets that sustain interest by flashing or squeaking, making a target 'appear' in a new position of gaze to induce refixation rather than following. Practical training and supervised practice are needed to achieve competence and should be part of primary care training schedules; orthoptists could make a valuable contribution.

\section{Visual acuity}

OPTOMETRIC FACTORS

The standard measurement of visual acuity uses a linear display of Snellen optotypes with specified spacing between each. Simply expressed each optotype is based upon a five by five square with the width of each component equal to one fifth and spacing between optotypes to four fifths of one square (figure). Any variations introduce optometric errors and a glance at Snellen charts available for adults reveals several in every chart! The size $60 \mathrm{~m}$ letter is single and considerable variation in spacing exists between other levels. The errors are even greater in tests designed for children; optotypes may be single at every level (Stycar, Sheridan Gardiner) or widely spaced at the critical levels in charts (Sheridan Gardiner 7 letter chart); some use pictograms with very varied integral proportions instead of letters. The idea was to simplify the cognitive aspects of the task but unfortunately considerable optometric accuracy was sacrificed for relatively little developmental gain.

Several recently designed tests, the Sonksen Silver acuity system (SSAS), the LH symbol system, and the Cambridge crowding cards (CCC) have addressed the optometric problem and present standard optotypes in correctly spaced linear display. $\int$ These rather than the previously mentioned tests should be used in diagnostic and surveillance work because they provide a measure meaningful in terms of the international standard, are more likely to identify children with clinically important minor errors, and to categorise the visual problem correctly in terms of educational impact in those with severe visual impairment.

Obviously, individuals with perfect sight will see at least $6 / 6$ on all test displays (standard linear, widely spaced linear, and single optotype). However, those with linear acuities of $6 / 12$ may well achieve $6 / 6$ or $6 / 9$ on the latter two resulting in loss of an opportunity for correction

§ The CCC uses ‘surrounded' optotypes. 
or preventative intervention. Personal experience of these measures in several hundred children with severe visual impairment suggests that the discrepancies in them are often even greater. The latter two types of measure may lull parents and professionals into non-recognition of the existence or degree of a child's difficulties in the classroom. Misinterpretation of visual difficulty as behavioural or learning disability and lack of appropriate educational support can then be emotionally as well as educationally damaging.

Children, like adults, function most successfully in situations and with material that they see 'at a glance'. Zealous encouragement by testers to 'look' or 'try' harder is perhaps not always wise; it may boost the measure through intelligent guess work because test displays designed for children embrace a choice of five or six rather than 26; the easiest way to clarify whether a child's problem is visual or behavioural is to return to a larger display or to step a metre closer. Leaning forward and peering are other clues in favour of a difficulty being visual.

The SSAS and the LH test have near test charts to standard specification. In children with severe visual impairment near as well as distance measures are essential for optimal prescription of spectacles, optical aids, and print size. In surveillance practice the distance measure is the crucial one because only $0.5 \%$ of children with normal distance acuity have an error of near." This advice is supported by a current study of 4-9 year old school children in Harrow using the SSAS. * The yield of visual errors from the distance measure was not increased by the addition of a near measure.

\section{DEVELOPMENTAL FACTORS}

There are five major interactive developmental issues of relevance to optotype tests in the preschool years: the maturation of linear acuity, the developmental benefit of single optotypes, the ability to match or name optotypes (letters, geometric shapes, pictograms), the effect of distance upon the ability to carry out the test task, and the acceptance of occlusion.

The body of evidence suggests that acuity for linear displays does not mature to $6 / 6$ or $3 / 3$ until the age of 5 years in some children. This is borne out by the preliminary analysis of a recent survey of 2 to 6 year olds in the community using the SSAS. $\dagger$ Although the adult norm of $3 / 3$ was achieved by $20 \%$ of 2,5 to 2,11 year olds, it had not been by up to $60 \%$ of 3,0 to 4,5 year olds and at least $20 \%$ of 4,6 to 6,0 year olds. The prevalence of minor visual errors in this age range is $6-12 \% .{ }^{1213}$ Twelve per cent of the youngest, $11 \%$ of the middle, and $1 \%$ of the oldest age group had acuities of $3 / 6$ or worse. In the context of prevalence and surveillance it would seem wise to refer children in the two younger groups with acuities of $3 / 6$ or worse and in the older group with $3 / 4.5$ or worse when testing with the SSAS. The findings of the Harrow study confirm $3 / 4.5$ as the appropriate level for referral of 6 to 9 year olds. *

Egan showed that there was little developmental advantage in using single optotype even with children under 3 years old ${ }^{14}$; this method therefore should only be used by specialist teams who appreciate its limitations.

The proportion of children able to match optotypes increases with age. The design of pictograms is often more complicated than that of simple letters or basic geometric shapes, making the first the most difficult to match. Pictograms are symbolic representations of objects and are more difficult than real objects or realistic life sized pictures to name and the skill to do so does not develop any younger; thus they offer no developmental benefit and in view of earlier comments about their optometric disadvantages there is little to advocate their use. Just over $80 \%$ of 2,5 to 2,11 year olds and over $90 \%$ of 3,0 to 3,5 year olds can match letters using the Sonksen Silver key card and training booklet. + This is a considerably higher proportion than Egan found for comparable age groups using the Sheridan Gardiner 5 or 7 letter key card ( $34 \%$ and $77 \%$ respectively). ${ }^{14}$ Egan had noted that some of those who had failed to do so achieved with $3 \mathrm{~cm}$ high plastic letters. Large $(18 \mathrm{~m})$ size letters for key card and training booklet was one of the developmental design features of the SSAS and probably accounts for the improved performance of the children in the SSAS study. $\dagger$ Although some 4 year olds can name letters, $32 \%$ of 5 year olds and $9 \%$ of 6 year olds are still unable to do so.*

The standard test distance for adults is 6 metres. Primary health care, school, and hospital clinic rooms rarely accommodate this requirement; corridors and large busy rooms are too distracting for young children and mirrors confuse them. In addition to difficulties with maintenance of attention, distance adds another cognitive dimension to the test task. Experience suggests that it is the proportion of children in each age band who are on the threshold of matching, that is those who have required the most training to do so, who are unable to continue once the examiner retreats to the test distance. The SSAS study that used a 3 metre test distance supports this view; the phenomenon being observed in about $22 \%$ of 2,5 to 2,11 year olds but only $3 \%$ of 3,6 to 4,0 year olds. $f$ There are no published figures but experience suggests that the proportion is greater when the test distance is increased to six metres. A three metre test distance is therefore advised for this age group. The productivity statistics in the Harrow study support this distance for primary schoolchildren also. *

In order to detect amblyopia and conditions (anisometropia, hypermetropia, and squint) that lead to it, testing each eye separately has always been advised. Unfortunately this requires occlusion which, as mentioned earlier, poses difficulties throughout the preschool era. Individual testers use different methods; spectacle frames with either the right or the left lens blackened or a loosely crumpled tissue held in the mother's hand have proved the most effective in our unit. Although, like the ability to match letters, acceptance of occlusion steadily increases with age some $10 \%$ reject it at 4 years. A small proportion of children who complete a both eyes open test, accept a patch and then find the matching task more difficult, further reducing 
the proportion in whom an each eye separately measure can be obtained. For surveillance, a test needs to be achievable in at least $90 \%$. This does not pertain until at least 4 years of age for each eye separately. However a both eyes open measure is obtainable with the SSAS in this proportion of 3 year olds. + Ninety six per cent of children have equal acuity or no more than one line difference between the eyes. "In approximately one third of the $4 \%$ with more than one line difference vision is not perfect in the better eye. The minute loss in reliability that results from testing preschoolers with both eyes open is outweighed by the gain in numbers who can be tested successfully. In the Harrow study no difficulty was experienced with occlusion of 5 to 9 year olds and the productivity statistics of the SSAS at three metres were improved by testing each eye separately. ${ }^{\star}$ Testing each eye separately is therefore recommended for this older age group.

While community paediatricians debate whether measurement of acuity should be part of preschool surveillance practice, it should certainly be attempted in any young child in whom vision is a concern.

\section{ALTERNATIVE METHODS}

Optotype tests take between four and 12 minutes to administer to preschool children and this makes their use in surveillance expensive of professional time. ${ }^{\star} t^{13}$ Clinicians require an alternative that is more developmentally appropriate, simpler and quicker to administer, and cheaper to produce. Objective measures, such as evoked response and preferential looking techniques, are unlikely to fulfil these criteria in the foreseeable future. The Sonksen picture test (SPT) may be a step in the right direction. ${ }^{15}$ Identification of photorealistic coloured pictures is dependent upon acuity; six pictures of everyday items are presented from three metres for a child with both eyes open to name/gesture. The SPT was completed by $98 \%(n=842)$ of 21 to 60 month old children in a mean time of less than two minutes; it identified seven of nine children with unequal vision and its productivity statistics for refractive error (sensitivity $65 \%$, specificity $47 \%$, and positive predictive value $36 \%$ ) compare favourably with the few other tests that have been subjected to evaluation. ${ }^{716}$ The SPT in its present form will be available shortly; the method is currently being refined.

Young children with severe visual impairment and those who are multiply disabled require more than a measure of acuity. Vision for a variety of learning materials (pictures, writing materials, communication boards, computer screens, etc) should be assessed. Methods are available, ${ }^{3817}$ the findings can be used both to prescribe the most appropriate visual environment for a disabled child and to monitor the effectiveness of low vision aids. ${ }^{918}$

1 Fraiberg S. Insights from the blind. London: Souvenir Press, 1977.

2 Sonksen PM. Vision and early development. In: Wybar K, Taylor D, eds. Pediatric ophthalmology; current aspects. New York: Marcel Dekker, 1983: 85-95.

3 Sonksen PM, Petrie A, Drew KJ. Promotion of visual development in severely visually impaired babies; evaluation of a developmentally based programme. Dev Med Child Neurol 1991; 33: 320-35.

4 Sonksen PM, Levitt SL, Kitzinger M. Identification of constraints acting on motor development in young visually constraints acting on motor development in young visually disabled children and principles

5 Hall DMB, ed. Health for all children. A programme for child health surveillance. Oxford: Oxford University Press, 1991.

6 Sonksen PM, Kingsley MPL. Visual disorders: severe visual impairment. In: Harvey D, Miles $M$, Smyth D, eds. Community paediatrics. Oxford: Butterworth Heinmann, 1992.

7 Hall SM, Pugh AG, Hall DMB. Vision screening in the under5s'. BMF 1988; 285: 1096-8.

8 Sonksen PM. The assessment of 'vision for development' in severely visually handicapped babies. Acta Ophthalmol (Copenh) 1983; 157 (suppl): 82-92.

9 Sonksen PM, Stiff B. Show me what my friends can see; a developmental guide for parents of babies with severely impaired developmental guide for parents of babies with severely impaired sight and their professional advisers. London: The Wolfson Centre, 1991 .

10 Fielder AR. The management of squint. Arch Dis Child 1989; 64: 413-8.

11 Alberman ED, Butler NR, Sheridan MD. Visual acuity of a national sample (1958 cohort) at 7 years. Dev Med Child Neurol 1971; 13: 9-14.

12 Kohler L, Stigmar G. Vision screening of four year old children. Acta Paediatr Scand 1973; 62: 17-27.

13 Ingram RM, Holland WW, Walker C, Wilson JM, Arnold PE, Dally S. Screening for visual defects in preschool children. Br F Ophthalmol 1986; 70: 16-21.

14 Egan DF, Brown R. Vision testing of young children in the age range 18 months to $4 \frac{1}{2}$ years. Child Care Health Dev 1984; 10: $381-90$

15 Hodes DT, Sonksen PM, McKee M. Evaluation of the Sonksen picture test for detection of minor visual problems in the surveillance of preschool children. Submitted to Dev Med Child Neurol (in press).

16 Rona RJ, Reynolds A, Allsop M, Morris RW, Morgan M, Mandalia $S$. Audit from preschool developmental surveillance of vision, hearing and language referrals. Arch Dis Child 1991; 66: 921-6.

17 Sonksen PM, Macrae AJ. Vision for coloured pictures at different acuities: the Sonksen picture guide to visual different acuities: the Sonksen picture guide to
function. Dev Med Child Neurol 1987; 29: 337-47.

18 Ritchie JP, Sonksen PM, Gould E. Low vision aids for preschool children. Dev Med Child Neurol 1989; 31: 509-19.

^Jayatunga R, Sonksen PM, Bhide A, Wade J. Measures of acuity in primary school children and their ability to detect minor errors of vision. (In preparation.)

† Salt A, Sonksen PM, Wade A. Maturation of visual acuity and compliance with the Sonksen Silver acuity system in young children. (In preparation.)

Sources of tests referred to in text

Sonksen Silver acuity system. Keeler (Optical) Ltd, Windsor, Berkshire.

Cambridge crowding cards. Clement Clarke International Ltd, London.

LH symbol test system. Lighthouse Low Vision Products, New York.

Sheridan Gardiner 7 letter test (chart and single optotype). Keeler (Optical) Ltd, Windsor, Berkshire.

Sonksen picture test. Inquire from author. 